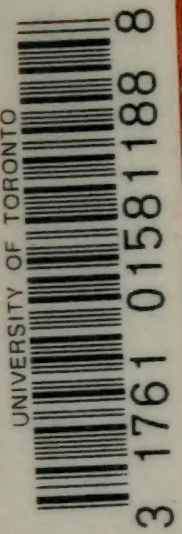





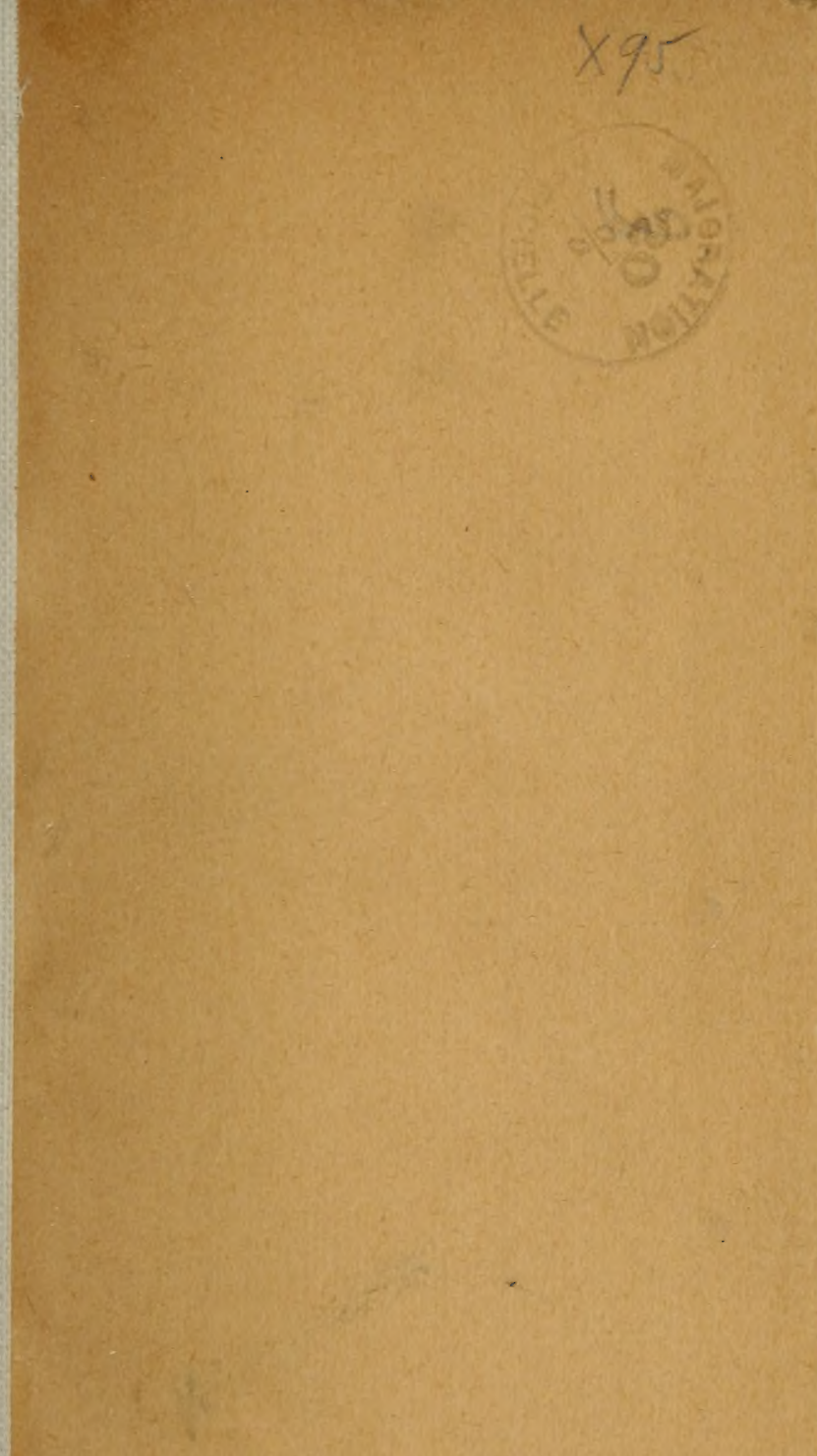




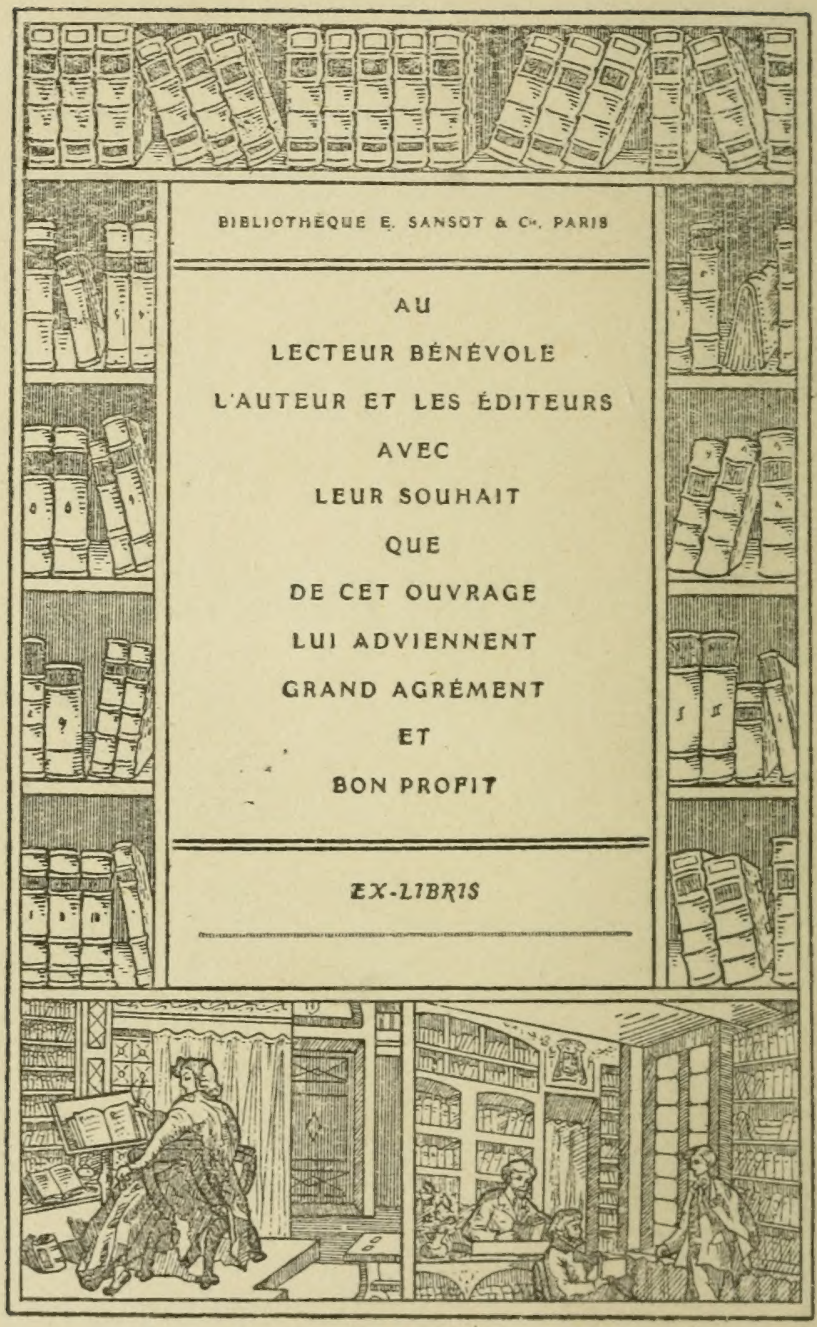




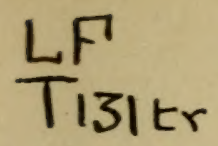

PETITE COLLECTION "SCRIPTA BREVIA"

\section{LAURENT TAILHADE}

\section{Le Troupeau}

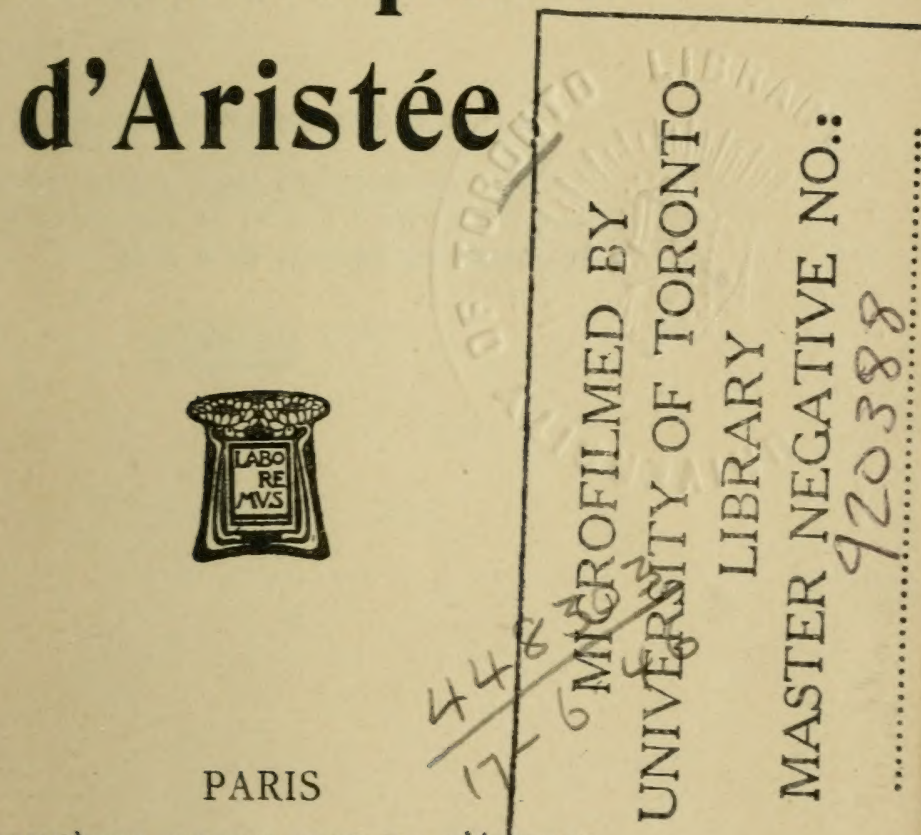

BIBLIOTHĖQUE INTERNATIONALE D'ÉLITION

$$
\text { E. SANSOT et Cie }
$$

7, RUE DE L'ÉPERON, 7.

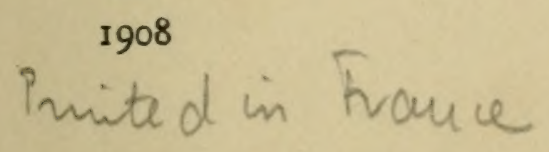


IL A ÉTÉ TIRÉ DE CET OUVRAGE :

Huit exemplaires sur japon impérial numérotés de 1 à 8 , et 15 exemplaires sur hollande, numérotés de 9 à 23 .

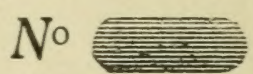




\section{A \\ M. ALIN CAILLAS \\ AU COLLABORATEUR}

$$
\text { ET A L'AMI }
$$

L. T.

Paris, le 21 Juin 1908. 

LE

\section{TROUPEAU D'ARISTÉE}

Le bachelier qui, frais émoulu des études classiques, arrive aux champs et n'a, pour cultiver l'apier de son domaine, d'autre enseignement que les prescriptions des Géorgiques, s'expose à la même disgrâce que le pasteur de Tempé. Il risque fort de voir, comme Aristaeus, les mouches qu'il possède fondre plus vite que la neige au mois d'avril.

Non que Virgile, observateur attendri et minutieux, ait omis, dans ce noble poème, quelque chose des leçons qu'un apiculteur informé pouvait donner aux hommes de son 
temps. Mais l'élevage des abeilles s'est transformé, a subi de telles et si ingénieuses modifications; il a, depuis un quart de siècle, renouvelé ses méthodes avec un tel esprit scientifique et des procédés à la fois si commodes et si pertinents, que la doctrine des vieux sages semble enfantine et pédantesque au regard des travaux dont s'honorent les modernes apiculteurs.

Néanmoins, la demeure, le site des ruches, les plantes dont il faut les entourer, les ennemis qu'elles redoutent: lézard, guêpe, mésange et Procné, dont la poitrine

fait les empreintes voir de doigts ensanglantés,

y sont déduits avec une certitude judicieuse; tous les conseils qu'il donne sur ce point ont gardé leur valeur intégrale. Mais les ruches ne sont plus tressées de vîmes flexibles ou d'écorces enchevêtrées; 
on ne détruit plus les essaims pour acquérir leur miel. Aux procédés barbares on a substitué des moyens efficaces et doux qui permettent d'enlever aux abeilles le superflu de leurs trésors, tout en leur faisant la vie plus confortable et mieux gardée.

Trois siècles après Virgile, Ambroise, évêque de Milan, écrivit, dans le $5^{e}$ livre de son Hexameron, un traité des abeilles et de leur merveilleuse complexion.

Là, sont enregistrés avec une complaisance que rien ne fait broncher les contes à dormir debout émis par l'antiquité au sujet des abeilles.

Quiconque a effleuré les études classiques est familiarisé avec le $4^{\mathrm{e}}$ chant des Géorgiques. Les cancres eux-mêmes, au cours de leurs études, ont effleuré, sinon dans le texte, au moins dans les pâles traductions des sorbonagres, l'épisode 
sublime d'Aristée, ces vers d'une incomparable harmonie et d'un charme si profond qui donnèrent à Glück, non seulement le sujet, mais la couleur de son Orphée. J'ose dire que j'en ai moi-même exécuté plusieurs copies en manière de pensums, à l'âge heureux où les divers cuistres préposés à l'instruction de la jeunesse nous transmettent le dégoùt des chefs-d'œuvre anciens, que j'ai honnêtement ravaudé les Nymphes de Cyrène, et la plainte d'Eurydice, et le vieillard Protée, au milieu de ses phoques.

Saint Ambroise est moins connu.

Il fut cependant, au IVe siècle, le plus haut représentant de la pensće chrétienne. Il préconisait la liberté de conscience à la façon des érêques modernes. Sa lutte pour la Victoire du Capitole contre Symmaque montre à quel point l'into- 
lérance - déjà ! - faisait partie des mours ecclésiastiques.

Ambroise néanmoins avait l'âme romaine. Il fut, autant et plus que Stilicon, le défenseur de la cité latine contre l'invasion des Barbares. Supérieur à son milieu par l'intelligence et par le caractère, il domina de toute sa hauteur un monde à l'agonie. Il eut, ̀̀ -la façon des forts, un amour très véridique de la douceur et de la paix. Comme toutes les âmes nobles, il aima la musique; c'est le plus harmonieux et le plus mesuré des Pères latins. Il fut orateur, musicien, poète; c'est de lui que procèdent le chant ambrosien et les chœursalternés dans la liturgie catholique. Son éloquence était pleine de suavité.

Le diacre Paulin raconte qu'un essaim d'abeilles vint se poser sur ses lèvres d'enfant, un jour que sa 
mère l'avait exposé au milieu des fleurs dans son berceau.

L'Hexaméron d'Ambroise est comme un abrégé d'histoire naturelle, un résumé de Pline le Jeune accommodé au goût chrétien. Nous y retrouvons de Virgile et de ses prédécesseurs les assertions bizarres à propos des abeilles. Néanmoins il ne mentionne pas la génération spontanée et même hétérodoxe dans les flancs d'un taureau en putrétaction.

M. Maurice Mrterlinck, que Mirbeau nommait autrefois le "Shakespeare de la Belgique "), rêva peutêtre aussi d'en être le Virgile. Dans le printemps de rgor, il donna 300 pages qui font bonne figure à côté de Michelet et du divin Mantouan.

Ses connaissances en matière d'apiculture sont infinies. Il a tout lu, depuis Aristote, Varron et Colu- 
melle, jusqu'aux tracts élémentaires et pratiques des Anglais, des Allemands, des Suisses, des Yankees, en passant par François Huber, Réaumur et Swammerdam.

C'est un grand écrivain, de qui les tableaux magnifiques et précis ont le nombre des plus beaux poèmes et l'exactitude minutieuse d'un manuel Roret. Le premier, il a écrit sur l'abeille moderne dans une autre langue que le patois melliffu en honneur chez les curés de village et les ministres huguenots dévoués à la culture apicole.

Ces recommandables pasteurs d'hommes et d'abeilles, assez mal à propos, agrémentent leurs dissertations techniques d'homélies et de maximes pieuses. Ils admirent les "desseins de la Providence " dans leur vermine ailée. Mais ils ont l'avantage d'offrir au jardinier rustique, à la fermière inculte, des com- 
pendium dont le langage est à l'unisson intellectuel des esprits le plus obtus.

" L'histoire de l'abeille, dit Maurice Mrterlinck, ne commence qu'au XVIIe siècle, avec les découvertes du Hollandais Swammerdam. ")

Michelet a conté la passion du grand naturaliste, inventeur du microscope, mort à 43 ans, épuisé moins par le travail que par la misère.

La Bible de la nature, son grand ouvrage, fut traduit en latin par les soins de Bœrhave, dans la première moitié du XVIII siècle.

Vint ensuite Réaumur. Puis, en I770, François Huber, de Genève, le sublime aveugle, qui pénétra plus avant que tout autre dans les secrets de l'apier et " qui reste encore le maître, le classique de la science apicole "). 
Si vous consultez le premier répertoire venu, encyclopédie ou lexique, vous apprendrez que l'abeille est un genre d'insectes appartenant à l'ordre des hymenoptères, section des porte-aiguillons, famille des apides sociales. Ce sont les "mellifères " de Latreille, les "mélites " de Duméril, les " avettes » de Ronsard et les " mouches à miel " du langage vulgaire.

Outre l'abeille domestique, cette riche famille ailée comprend les bourdons, les psytires, les mélipones, les trigones, sans compter les variétés d'Amérique et " le petit peuple des rochers, les abeilles noires sauvages de l'Inde, toujours affairées et furieuses ", qui règnent sur la jungle au point que le tigre ou l'éléphant s'écartent de leur chemin (Rudyard Kipling, Chien rouge).

Les bestioles que cultivent Maurice Mæeterlinck et l'abbé Duques- 
nois se montrent de complexion moins farouche.

Quand elles frappent, c'est pour défendre leur vie ou leur postérité contre un danger, tantôt réel, tantôt imaginaire. Elles font à la Cité, à la République, aux enfants à venir, le sacrifice de leur personne; elles recommencent les dévouements d'Elius Tubero ou de Mucius Screvola, sachant qu'elles ne survivront pas à leur coup de poignard, que la blessure de l'adversaire entraînera leur mort, douloureuse et certaine.

Barbelé, en forme d'hameçon, leur poignard pénètre dans la plaie, y porte l'acide formique et sa brûlure vésicante, mais il n'en peut sortir. Il faut, par un arrachement brutal qui enlève une partie de son arrière-train, que l'insecte abandonne l'arme empoisonnée au plus profond de la blessure pour, 
à quelques pas plus loin, mourir dans l'aisselle d'une feuille ou le calice d'une Heur.

Un poète a célébré " leurs ailes d'or » et « leurs flèches de flamme ».

Ces flammes vives ont, comme celles de la cantharide, et suivant la dose, une action néfaste ou curative sur l'organisme humain. Les piqûres d'abeilles, après la révulsion cruelle du premier moment, procurent aux goutteux, aux arthritiques, un bien-être que les médecins préconisent à l'égal des sources les plus vantées.

Le formol, qui joue un rôle si notable dans la thérapeutique moderne, agit avec une puissance décuplée alors qu'il est fourni, élaboré par un organisme vivant; de même, les eaux thermales, supérieures mille fois aux produits obtenus par synthèse dans le but de les remplacer. 
Qui n'a vu, près des fermes de Bretagne, sous le couvert des pommiers; qui n'a vu, parmi les fleurs odorantes et sauvages des Pyrénées, adossée aux murs de schiste et d'ardoise où la guêpe suspend son nid pareil à une fleur de papier bleu turquin, la ruche conique, la ruche virgilienne, d'osier, de troène, de lattes ou de paille? Les gâteaux y sont fixés par le soin même des abeilles. C'est le type archaïque, dont la routine a si longtemps prévalu et dont l'incommodité faisait la récolte à la fois plus dangereuse et moins rémunératrice.

L'apiculture moderne substitue à ces cloches anciennes des huttes spacieuses, aérées, commodes aussi bien pour le travail que pour la collecte de la cire et du miel. Ruche universelle, ruche Layens, ruche à sections américaines, ruche de l'abbé Sagot, toutes se construisent sur 
la même donnée, à savoir : que le toit de la ruche doit être mobile, ainsi que les cadres ou rayons destinés à recevoir le gâteau de miel.

On peut voir, à l'automne, chez la plupart des marchands de comestibles, ces rayons d'un beau blanc crémeux, qui renferment la nourriture parfaite, élixir de la vie animale et de la vie florale. Plus hardis que les végétariens d'à présent, les Sages de la Grèce osaient substituer le miel aux aliments épais qui sustentent le commun des hommes.

La récolte du miel, d'empirique, est devenue scientifique. Un propriétaire de ruches calcule, d'après la température de l'été, d'après la floraison plus ou moins riche de telle ou telle essence végétale, d'après la vigueur des essaims et le poids des ruches, le rendementexact de ses abeilles. Il n'ignore pas que telle ou telle fleur donne 
un miel plus ou moins pur, que les acacias et le sainfoin du mois de de juin fournissent le produit de luxe, tandisquele miel jaune foncé recueilli sur la fleur de sarrazin, ne vaut guère, sinon pour fabriquer le pain d'épice. Il n'a garde d'ignorer que certaines fleurs communiquent un arôme désagréable ou trop spécial, qu'il faut aimer beaucoup le parfum du tilleul pour se plaire au miel butiné dans sa fleur, que le miel du châtaignier manifeste un goût désagréable et que celui que donne le vernis du Japon (ailante) exhale une odeur absolument nauséabonde.

Les mœurs des abeilles ont, de Virgile à Mrterlinck, prèté aux variations les plus magnifiques, aux traits des moralistes, aux descriptions enthousiastes des amateurs de plein air.

Cependant, et malgré leur amour 
du paysage, les Romantiques paraissent les avoir méconnues.

Lamartine, çà et là, nomme « les abeilles de l'Hymette ", qu'il semble tenir pour des animaux fabuleux ou tout au moins allégoriques, ayant leur place dans la langue élégiaque au même titre que l'aigle, le cygne et autres animaux de style soutenu.

Victor Hugo, bien qu'il témoigne des environs de Paris une vision si juste et si pittoresque, ne semble avoir pris connaissance des mouches à miel que pour les chasser du manteau impérial.

Musset n'avait, en fait d'animaux, connu que les kingcharles et les perruches vertes en honneur chez $M^{\text {mes }}$ de Bauséant ou de Langeais.

Seul, parmi ses contemporains, Michelet eut l'horreur de l'à peu près. Quand le goût lui vint d'étu- 
dier les sciences naturelles, pour se reposer d'avoir cheminé si longtemps les routes poudreuses de l'Histoire, il appliqua les mêmes procédés, la même conscience d'investigation; il regarda la Nature comme il avait autrefois regardé les archives. Son livre des abeilles est, à coup sûr, le meilleur de l'Insecte. Il vulgarise la doctrine des savants; il célèbre en poète le geste des infiniment petits qui montrent l'exemple des vertus civiques, de l'abnégation, du dérouement, du sacrifice perpétuel que l'Individu fait de soimême à la Collectivité.

Dans Gulliver, Swift, réduisant à la taille des insectes l'ambition, la noirceur et la bêtise humaines, en donne la plus cuisante satire. Ne pourrait-on affirmer, par contraste, que la ruche est une sorte de Lilliput à rebours dont les infimes citoyens appremnent au " roi des 
animaux " le travail, la concorde et l'amour de la paix?

Quels sont les fondateurs, les appuis, les dignitaires, les ouvriers de cette République irénienne,dont le calme, la vertu, les sages institutions, l'esprit de labeur et de concorde montrentà l'humaine inquiétude un si touchant exemple et de si fermes leçons?

Quel pasteur de peuples a défini les bornes de la Cité? Quel dieu en a promulgué les lois? Quel sénat, quels tribunaux maintiennent l'ordre, imposent à chaquecitoyen le dévouement le plus absolu, un dévouement de toutes les heures, à la tâche collective?

L'apier forme une sorte de couvent industriel et militaire où les vierges qui produisent le miel se consacrent dès l'enfance au travail, au renoncement et à la chasteté.

Esclaves, par le dur métier 
qu'elles exercent, mais reines sans conteste de la ruche opulente où, dans leurs berceaux ambrés, dorment les essaims de l'avenir, elles observent la dure obédience qu'impose l'amour des fleurs et l'orgueil d'élaborer leurs nectars.

Trois groupes d'individus composent l'effectif de la ruche : ce sont les reines, les mâles ou faux bourdons, et les ouvrières, asexuées dès leur berceau.

La reine, qu'une longue erreur, transmise par l'antiquité, fit longtemps prendre pour un chef, pour un roi, pour un dynaste guerrier, pour une sorte d'Agamemnon à six pattes, au corselet brillant, n'a d'autre besogne que d'enfanter le peuple et de faire couler dans les alvéoles prêtes le ruisseau de fécondité qui jaillit de ses flancs.

Ce « roi », chanté par Virgile, saint Ambroise l'a vanté pour des raisons 
moins prégnantes aujourd'hui. Ce qu'il admireavant toutchez les « avettes) pour employer la langue de Ronsard, c'est la parthénogénèse, le fait de se reproduire sans avoir recours au stratagème que Daphnis apprend de Lycenion.

- "Silence! arrêtez-vous, dit WWagner, le famulus du docteur Faust, à Méphistophélès.

- Qu'y a-t-il ?

- Un homme va se faire.

- Un homme? Vous avez donc enfermé des amants quelque part.

- Bon! dit Wagner. Une femme et un homme, n'est-ce pas? C'était l'ancienne méthode. Nous avons trouvé mieux. "

Saint Ambroise ne parle pas autrement.

"Ce communisme est grand, - dit-il, - mais combien plus chez les abeilles que chez les hommes! Chez les abeilles qui, seules de tous les êtres animés, ont une postérité commune, hantent la même demeure et bornent à un seuil unique la limite de leur patrie, les abeilles pour qui toute chose 
est indivise, travail, nourriture, entreprise, usage et fruit; les abeilles, de qui le vol même appartient à la communauté.

" Que dirai-je de plus ? La génération appartient a la République. L'intégrité de leur corps virginal est aussi commune à tous les citoyens, puisqu'ils ne s'unissent par aucune étreinte, puisqu'îls ne sont terrassés ni par le désir, ni par les maux de la partu. rition, puisqu'ils émettent spontanément les essaims adultes que leur bouche a butinés dans les feuillages et les herbes".

Ambroise avait assisté à la grandeur, puis à la chûte d'Eutrope. L'air vibrait encore des invectives de Claudien et des adjurations de Chrysostôme. Il félicite les abeilles de donner la couronne au plus digne, au Roi formé par la nature, et de ne pas instaurer au pouvoir les eunuques plus soucieux de leurs bénéfices que du bien de l'Etat. Car il ignore que toute la politique du rucher est précisément fondée sur l'eunuchisme comme celle des Empereurs byzantins. 
Grande, mince, plus fine et plus forte que les mâles et que les obscures travailleuses, la reine-mère des abeilles est construite en longueur, haute sur ses pattes, comme tous les animaux de race, comme le cheval de course ou le rossignol.

Plus tard, déformée par la maternité, son ventre acquerra des proportions monstrueuses, la retiendra prisonnière dans la ruche, alors même que sa garde d'honneur, toujours attentive et présente, ne serait pas là pour neutraliser son inconstance et la ramener au devoir.

Mais, telle qu'on l'observe d'abord dans sa parure de fiancée, éclatante d'une chaude couleur brune et déployant aux souffles de l'avril ses ailes virginales, c'est un des plus beaux insectes dont s'émerveillent les regards humains.

Maurice Mreterlinck a célebré les noces de l'abeille, son vol nup- 
tial à travers les parfums, dans l'azur du mois de mai, la communion de tout un peuple avec l'amour que sa royale enfant va connaître pour lui.

Passagères délices! Le vol qui porte la mère du peuple dans le libre espace ne dure qu'un instant.

Désormais féconde, la reine appartient à la Cité. Après la brève et joyeuse étreinte de l'hymen, voici venir pour elle une ère de maternité claustrale qui ne finira qu'avec ses jours. Dès qu'elle a pris contact avec son débile époux elle regagne pour n'en plus sortir sa prison de topaze et d'or.

Les faux bourdons tiennent dans la ruche le même emploi que les princes consorts dans les états où la loi salique n'est pas en vigueur. Ils jouissent de quelques-unes des prérogatives décernées aux "étalons de la reine " par les peuples civi- 
lisés. Ilsgaspillent le trésor commun, s'occupent à des travaux ineptes et se gorgent de boissons enivrantes, comme s'ils avaient eu l'honneur d'épouser Wilhelmine. Ils sont presque aussi infatués d'euxmêmes qu'un gentleman à bonnes fortunes. L'oisiveté les engraisse et les enlaidit, leur profession étant de celles qui manquent un peu de gloire quand se montre la maturité.

Aussi, dès les premiers jours de septembre, aux approches de l'équinoxe, marquant le terme de l'année apicole qui est brève et dont l'activité ne s'étend guère que d'avril à octobre, quand les fleurs deviennent rares, quand

le dahlia met sa cocarde et le souci sa toque d'or,

les ouvrières en font un grand carnage. Elles transpercent de leurs aiguillons et rejettent hors de la demeure commune ces amants offi- 
ciels qui n'ont pu faire usage de leurs brillantes facultés, ces maris désormais superflus, qui grèvent la communauté sans lui fournir aucun apport.

L'ouvrière concentre en elle tout l'intérêt du rucher. Elle ne paie pas de mine. Cette mouche velue, aux couleurs ternes, au vol bas et comme appesanti du lourd attirail qu'elle porte, n'a pas l'éclat des insectes mondains, papillons somptueux, éclatantes libellules.

Sa livrée est celle du travail qui crée et qui féconde; elle rappelle ces cotes, ces bourgerons de prolétaires, noirs de graisse, lourds de plâtre et tachés de poussières, que portent sur leurs membres robustes ceux qui enfantent la richesse et bâtissent des palais.

Ces vierges sages, dont, à partir du berceau, un aliment restreint atrophia les organes, assument tra- 
vaux, responsabilités, défense et maintien de l'ordre public.

Les cirières, aux chatons des sapins, aux bourgeons des peupliers, des bouleaux, des trembles et surtout des marronniers, demandent le propolis, la forte résine convenable aux gros ouvrages, aux murs de soutènement, aux assises du rûcher. Elles trouvent la cire dans les menues fleurs dont le nectaire comble de miel une autre catégorie d'ouvrières. Elles recueillent aussi le "pain des abeilles " sur les houppes dont leurs jambes sont pourvues et, dans leur vol fécondant, insèrent au pistil des fleurs femelles quelque chose de la poussière mâle tombée des étamines.

Elles fomentent les noces du jardin.

Quand elles sont approvisionnées de liquide sucré et qu'elles ont garni leur jabot avec la miellée 
prise dans les corolles, bien vite elles regagnent leur domaine pour dégorger la précieuse substance dans les alvéoles déjà préparées par les cirières.

Mais ce n'est pas uniquement le suc des fleurs qu'elles élaborent ainsi. Elle ajoutent au précieux liquide leur acide formique; ce liquide puissant donne au miel, dont il neutralise les ferments, quelque chose de son âpre vertu et ce goût fauve, cette " odeur de mouche " appréciée par tous les connaisseurs.

L'abondance de nourriture et l'ampleur du dortoir, où l'insecte, d'abord à l'état de larve, broute la miellée en attendant la métamorphose suprême, dort son sommeil de chrysalide, suffit pour lui conférer les stigmates de l'héritier présomptif, du prétendant à la royauté. 
Les ouvrières ont des cellules plus étroites, une provende moins généreuse, une bouillie de sevrage d'où le miel est à peu près absent.

Elles pondent quelquefois; c'est la parthénogénèse, la parturition virginale révélée, peu de temps après Huber, par le curé silésien Dzierzon de Carlsmark.

Ainsi, chez les abeilles, androgynes au début, la dépense de bouche, déterminant le sexe, établit la fonction.

Les trompes des truis sortes d'abeilles, par leur forme et leur dispositif, suffiraient à expliquer leur vie. Atrophiée ou presque, chez la Reine gorgée, dès avant sa naissance, de la pâtée royale, du savoureux mélange fait de pollen et de miel, on la voit se développer chez les mâles qui, dans leurs loisirs, butinent çà et là, font en amateurs des incursions à travers les prés et 
les jardins fleuris. Quand ils ont assez de leur couvert habituel, quand les prend un désir de vagabondage et de prétentaine, ils sont pourvus d'un outil qui leur permet de manger à même le nectaire des sainfoins ou des pommiers.

La trompe de l'ouvrière est un engin d'une autre envergure. Longue étrangement, si vous la compareż à la tête, elle peut fouiller jusque dans les recoins les plus secrets la chambre nuptiale des corolles, arracher le pollen aux étamines et, le transportant sur le pistil, présider aux échanges d'amour entre les plantes éloignées.

Il est aisé de voir l'économie admirable de cet organe. Une image fortement grossie en donne le détail, montre les rudes soies qui le hérissent, assez comparables aux brosses dont les fumeurs de pipes se servent pour entretenir leur ca- 
lumet. Ces soies irritantes accrochent les fertiles poussières, les transportent de calice en calice, paient aux fleurs solitaires la rançon du miel en fécondité.

La configuration de l'abeille tout entière porte au plus haut degréce caractère de spécialisation.

Ouvrez attentivement le corps de la bestiole; regardez à la loupe ce microcosme, cet univers en raccourci que dévoile aux regards le plus humble des êtres organisés.

La structure de l'abeille, avec ses dix mille paires d'yeux presbytes et ses trois ocelles myopes implantés sur le front comme l'œil unique de Polyphème, avec sa tête, son thorax, son abdomen dont les stigmates s'ouvrent à l'air du dehors (car l'insecte respire par le flanc), est à peu de chose près semblable à l'anatomie des autres hyménop- 
tères, de la guêpe, sa sœur et de la mouche, sa cousine.

Mais l'appareil digestif, laboratoire du miel, està peu près unique dans l'anatomie des insectes.

Voici, dominant l'estomac proprement dit et les intestins lovés en méandres, voici le jabot, assez comparable au deuxième estomac des ruminants. C'est la pièce antérieure dans laquelle tombe directement le nectar à peine dégluti. Il y subit une transformation immédiate, se dédouble en lévulose et en glucose. Animé par l'acide formique, il prend la riche saveur, ce goût âpre et délicieux qui fait du miel un incomparable aliment.

Enfin les anneaux de l'abdomen, par un mécanisme fort simple, rejettent la cire liquide, que l'insecte pétrit en boulette, puis élabore avec ses fortes mandibules pour en former les rayons. 
("L'abeille est en sueur », disent les profanes, quand tout entière elle ruisselle des matériaux fluides encore qui serviront à édifier les murs, le Capitole des infiniment petits.

La patte de l'insecte ne manifeste pas une moindre adaptation à l'industrie.

Composée de trois articles: tharse, cuisse et jambe, elle porte à son extrémité des houpettes velues qui rappellent, tant par la qualité du poil que par son implantation, les brosses de la langue et correspondent aux mêmes utilités.

Dans les espèces florales où le pollen s'agglomère en pelote, il faut une intervention assez énergique pour le détacher. Toutes les plantes ne possèdent pas la faculté suprême dévolue au palmier de jeter aux souffles du désert une semence toujours féconde que le 
hasard seul peut faire proviguer.

Entre la cuisse et le tharse, la corbeille est employée à recueillir le pollen que l'ouvrière emportera dans la ruche.

Une vie active, en plein soleil, les pénibles travaux des champs, les âcres parfums qu'elles butinent, l'acide formique, principe et générateur de leur activité, donnent aux abeilles une soif inextinguible. Leur tempérament "aduste ", pour parler comme Michelet, fait qu'elles se plaisent aux fontaines, aux sources mystérieuses qui, parmi les caltas et les myosotis, jaillissent au fond des cressonnières.

Tantôt sur une feuille, tantôt sur un caillou branlant, elles se baignent au milieu des fleurs, sans craindre le destin de Narcisse ou d'Ophélie.

Ayant examiné sous un aspect général et cursif l'abeille indivi- 
duelle, entrons dans la Cité que régit une politique intelligente et sévère dont l'institution eût été accueillie avec faveur par les éphores de Lacédémone ou les compagnons philosophiques de l'Empereur Julien.

L'organe fait la fonction. Mais, chez les abeilles, le berceau crée l'organe, - on ne saurait trop insister sur ce point. La simple larve qui, dans une cellule ordinaire, nourrie de vivres plébéiens accomplit le cycle des métamorphoses pour, à l'état d'insecte parfait, assumer la qualité d'ouvrière, devient, suivant la mesure de son appartement et la vigueur de son alimentation, reine ou faux bourdon.

A côté des cellules vulgaires voici le dortoir des mâles, voici le dôme spacieux où l'aspirante à la royauté parcourt la première étape de sa vie et, comme une Belle-au-bois- 
dormant, passe les deux semaines de ténèbres nécessaires à sa formation. Le palais qui l'abrite a déjà la forme de son abdomen, de ce ventre sacré, objet du culte, de la vénération et de l'amour de tout un peuple.

Quand elle retourne dans la ruche, ensemencée et prolifique, ses dames d'honneur, ses ménines la conduisent dans les cadres béants sur les alvéoles désertes. Elle tourne en cercle, et, dans chacune, elle dépose un œuf, jusqu'au temps où, le cadre rempli et chacune des cellules operculées, ses gardiennes rigilantes la conduisent vers un autre quartier, qu'elle peuplera d'espoir et d'avenir.

L'homme intervient dans le pacte social des abeilles depuis le temps où les rois d'Israël goûtaient le miel sauvage à la cime des arbres, où le polythéisme hellénique peu- 
plait d'abeilles d'or la barbe de Dionysns, père de la vendange et des fruits savoureux.

La ruche fut d'abord logée au creux d'un arbre, dans l'infractuosité d'un rocher, sous des écorces mal jointes. Plus tard, le vannier assouplit en corbeilles les vîmes flexibles et les baguettes de l'osier. Quand les sculpteurs du IIe siècle déguisent le bel Antinoüs en berger d'Arcadie, ils ne manquent pas de le montrer à côté d'une ruche qui ressemble aux modèles employés encore dans les villages du sudouest.

L'école d'agriculture de Grignon, dont le rucher, administré par MM. Mamelle et Poujol, suscite l'admiration des visiteurs, garde, à titre de document, les modèles archaïques de l'ancien rucher.

La hutte primitive, coiffée d'un paillasson qui la préserve du froid 
et de la pluie, ainsi qu'en usent les jardiniers pour les plantes d'orangerie à qui l'on fait passer l'hiver en pleine terre, apparaît avec tous ses inconvénients; elle rappelle, dirait-on, les hôtels du vieux Paris, ces logis inconfortables que la mai son moderne, aérée et spacieuse, chasse peu à peu des quartiers civilisés.

Voici les huttes modernes: ruche horizontale de Layens, ruches $\mathrm{Da}$ dant. Avec leurs cadres mobiles, arec leurs portes, leurs tiroirs, ces demeures nouvelles permettent aux abeilles de donner au maitre, sans inquiétude ni danger, une récolte plantureuse, tandis que celui-ci respecte dans tous leurs droits les mouches aux œufs d'or qui fondent sa prospérité.

La mouche, en dépit de sa réserve professionnelle, semble néanmoins user quelquefois pour son propre 
compte des breuvages récoltés. Estce le soleil qui l'enivre ou bien les odeurs de ce premier printemps? La saison pleine de joie et de promesses ouvre les portes de l'année et couronne de timides fleurs l'adolescence de la terre.

Le temps n'est pas encore venu des églantines, Des parfums répandus dans les baisers du rent, Des raines échangeant leurs clansons argentines Avec le cri du merle et de l'engoulevent.

Mais les frênes déjà ravivent leurs squelettes Et chauffezt leurs bourgeons impatients du ciel, Les sylphes ont repris leur place aux violettes Et l'abeille commence à méditer son miel.

Mais dans chaque ravin du blane amphitheàtre, Une source aux bouvreuils a rouvert son miroir; Mis la nuée est blonde à l'horizon bleuâtre, Mais c'est mieux qu'un bonheur eacor : c'est un espoir!

Certaines abeilles dévastent les corymbes jaune pâle du mahonia, ces fleurettes qui " font le printemps ", pour parler comme Théophile Gautier. On n'en composerait pas un bouquet au mois de juin ; mais, quand elles s'épanouis- 


\section{IE TROUPEAU D'ARISTÉE}

sent, les corolles du mahonia attestent même aux gens qui n'ont pas lu Horace que l'âcre hiver se délie et que les zéphyrs sont de retour. Les orchidées indigènes et, nommément, le groupe des ophris, tiennent dans la féerie du mois de mai l'emploi des travestis. Aussi justement qu'une autre famille botanique, ces étranges fleurs pourraient assumer à bon droit le non de "personnées ". Porteuses de masques, elles imitent les hyménoptères communs dans nos pays arec un mimétisme surprenant.

D’après Darwin, ce carnaval des fleurs a le but le plus sérieux; par leur conformité avec le type de leur race, les ophris invitent mouches et papillons à se poser sur eux, à consommer l'hymen dont ils rêvent.

Ce sont les porte-insectes, frelon velu et mordoré, mouche 
importune et volage, abeille végétale prenant comme paranymphe l'insecte fraternel et, pour en célébrer la fête, lui tendant une coupe de nectar.

La concurrence vitale qui donne aux abeilles de généreux amis dans les tribus de la prairie ou du jardin leur suscite en même temps de sombres et redoutables ennemis.

Dans le climat tempéré de l'Europe occidentale croissent des plantes scélérates qui, pareilles à la dionée, aux saracenas de la zone tropicale, se repaissent de meurtre, des végétaux carnassiers dont la mort alimente les forces et l'éclat.

La drosère nommée, à cause des gouttes fraîches qui toujours emperlent son feuillage: " rosée du soleil ", rossolis, saisit les mouches et les digère. Sa "rosée " est un suc gastrique, acide après le repas, comme celui des mammifères, qui 
dissout les substances animales et prépare leur absorption par les organes de la plante.

Mais plus que tout autre végétal des prés ou des coteaux l'abeille redoute un gramen, l' " accrocheabeille ", qui se nomme, de son nom d'académie, sétaire rerticillée.

Ses panicules accrochent les pattes de l'insecte comme les mains des gnomes barbus qui, dans la forêt des légendes, saisissent au passage les vagabonds attardés.

Près des ennemis enracinés au sol qu'un peu de prudence et la mémoire des localités permet de fuir avec succès, l'abeille voit se dresser une autre armée, effrayante, d'adversaires ailés, griffus, pleins de vigueur et d'appétit:

L'homme, d'abord, l'étouffeur sinistre qui dilapide son propre bien et tue avec acharnement l'in- 
secte profitable, pour le plaisir de tuer;

Ensuite, le moineau plébéien et la noble hirondelle, tous deux friands de miel;

Puis, la tribu des insectes guerriers: libellules, demoiselles, qui, dans leur vol harmonieux, pourchassent la proie abondante l'insecte alourdi par un trop riche butin.

Le philante apivore, mouche qui volète sur les reines des prés, les flouves odorantes et autres plantes fourragères, le philante d'aspect modeste, sans avoir l'air de suivre un méchant dessein, dès qu'il aperçoit une abeille regagnant sa colonie après récolte faite, la poignarde gentiment et porte à sa larve qu'elle enterre l'abdomen rempli de miel.

Un autre bandit non moins redoutable, c'est le sphynx atropos, ainsi nommé à cause des stigmates 
qu'il porte sur son corselet et qui figurent assez exactement une tête de mort, comme le scarabie d'or d'Edgar Poë. L'Europe le doit à M. de Parmentier. Sa larve, en effet, vit dans les tubercules de la pomme de terre dont Louis XVI, comme chacun sait, portait une fleur à sa boutonnière.

Le sphynx atropos entre dans les ruches ainsi que dans une ville conquise. Il effondre les alvéoles, piétine le couvain; il se gorge de miel comme un électeur, de sandwiches, au bal de l'Hòtel de Ville.

Pareil aux guerriers de Guatimozin, il est couvert d'une armure qui le met à l'abri de la dague et du poison. L'acier de damas qui d'un revers tranche les têtes les plus fières s'émousse dans un édredon. L'aiguillon des avettes ne transperce pas la fourrure du monstre. Le brûlant acide formique 
glisse comme averse d'orage sur sa lourde toison.

Aussi, quel zèle pour garder les portes de la ville et dresser devant l'intrus des obstacles décisifs!

Les abeilles se pressent; des pattes, des mandibules, de tout leur être, elles s'efforcent de le saisir, de trouver dans sa cuirasse capitonnée une place vulnérable.

D'autres voleurs encore menacent la richesse de l'apier. Je nomme pour mémoire l'ours, car ce plantigrade est assez peu fréquent aux environs de Paris.

Viennent ensuite les divers rongeurs qui, s'il faut en croire la Batracomyomachie, ne redoutent que deux ennemis sur toute la terre, l'épervier et la belette, et aussi la ratière lamentable où veille une destinée pleine de ruse: la légion des souris, mulots, campagnols, voleurs de miettes, rongeurs de pain, 
troueurs de jambons et lécheurs de meule, dont les fortes dents déchiquètent le bois, ouvrent dans les murs de la cité des brèches funestes, pillards sans foi qui vivent du travail d'autrui et pour qui l'on prend avec raison à la lettre le $\mathrm{Sic}$ vos non vobis de l'inepte quintil imputé à Virgile.

Voici, l'oreille au vent, une jeune souris, un hasmter qui font des mines comme use élégante de province ou bien, "se glorifiant de leurs ventres ", grignotent un chanteau de fromage et méritent le beau nom de Tyroglyphos que n'eût pas manqué de leur décerner l'aveugle Homère.

Aussi, les mouches guerrières, les petites walkyries aux ailes d'or, comme elles fondent sur l'envahisseur et le transpercent de leurs glaives! Et, quand il est mis à mort, avec quelle prudence elles embau- 
ment le cadavre, qui bientôt empoisonnerait l'atmosphère et serait pour la République un mal pareil à ce fléau que les apiculteurs nomment, je crois, la "loque ", c'est-à-dire la décomposition du couvain!

a S'il est impossible d'expulser le rat ou la limace ou de les dépecer, les abeilles l'enferment méthodiquement et hermétiquement dans un véritable sépulcre de cire et de propolis, qui se dresse bizarrement parmi les monuments ordinaires de la cité (Les adverbes joints de M. Maurice Mrterlinck font admirablement). J'ai rencontré, lan dernier, - poursuit-il, dans une de mes ruches, une agglomération de trois de ces tombes, séparées comme les avéoles des rayons par des parois mitoyennes, de façon à économiser le plus de matière possible. Les prudentes ensevelisseuses les avaient élevées sur les restes de trois petits escargots qu'un enfant avait introduits dans leur phalanstère " (La Vie des Abeilles).

L'épée ennoblit ce qu'elle touche. La bête immonde mise à mort par le dérouement civique de l'abeille 
mérite d'être embaumée dans les aromates, comme un Pharaon, sous les monuments de la Cité victorieuse, de dormir éternellement au milieu des parfums dans un tombeau d'ambre, d'agathe et de cristal.

$$
* *
$$

Si l'on passe de l'Histoire naturelle au monde légendaire, le domaine de l'abeille s'agrandit. Les peuples indo-européens la font participer aux métamorphoses des dieux.

Elle butine sur la bouche des poètes le miel de la sagesse. Elle prête au jeune Eros, au Désir qui peuple les terres et les mers, son irrésistible aiguillon. Tantôt, sous les myrthes du cap Sunium et les cyprès d'Académus, elle cherche les lèvres de Platon; tantôt, amoureuse et légère, elle bourdonne, dans 
les vers de Méléagre, et, plus tard, se pose, au milieu des fleurs champêtres, sur le bouquet d'Antinoüs.

La Bible juive est comme une alvéole remplie de miel, toute bruissante d'ailes et de rumeurs guerrières.

Les textes abondent.

Jonathas, fils de Saül, est condamné à la mort pour avoir, un jour d'abstinence prescrite par le Roi, touché au miel des abeilles sauvages. Sa plainte douloureuse et charmante sert d'interprète à la mélancolie éternelle des êtres jeunes frappés dans leur matin. Elle symbolise cette amertume qui jaillit, dit Lucrèce, de la fontaine des plaisirs :

"Goûtant, jai goûté un peu de miel au bout de ma baguette, et voici que je meurs!»

Déborah (l'abeille), prophétesse et femme de Lapidoth, jugeait Israël. Elle résidait sous le "palmier de l'abeille », entre Rama et Beț- 
Hel, dans la montagne d'Ephraïm. Vers elle montaient, pour terminer leurs différends, les Bene-Israël. Deborah fut l'inspiratrice de Jahel qui, sur le Thabor, tua, dans sa tente même, le chananéen Sisara, généralissime du roi d'Ashur.

L'histoire de Samson éclate d'ironie et de férocité. Il n'en est pas de plus juive. Elle contient, comme la plupart des contes orientaux, une de ces propositions équivoques, de ces lemmes ambigus, oú se plut toujours la goguenarde subtilité d'Israël.

Près d'Ascalon, dans le bourg de Thamnata, gardant les vignes des Philistins, Samson aperçoit une fille étrangère. " Tu as blessé mon cœur d'un regard de tes yeux ", dit-il à cette jeune belle dont, malgré les objurgations paternelles, il ne tarde pas à demander la main. Tandis qu'il fait sa cour, il ren- 
contre, chemin faisant vers la demeure de sa fiancée, un lion de forte taille. Mais, soutenu par l'esprit d'Iaveh et sans rien avoir dans la main, il abat le fauve comme un chevreau.

Revenant, quelque temps après, chez la femme qui plut à ses regards, il se détourne pour voir ce qui reste du lion. Voici qu'un essaim d'abeilles a fait son miel dans la gueule du cadavre. Samson recueille ce miel et le mange en allant vers Thamnata.

Bientôt le festin des noces a lieu où trente jeunes hommes lui servent de paranymphes.

Samson leur dit:

- Je vais vous proposer une énigme. Si vous me la devinez pendant les sept jours du festin et que vous la résolviez, je vous donnerai trente tuniques de lin et treute habits de couleurs variées. 
- Propose ton énigme, lui répondent-ils, que nous l'entendions.

Et Samson leur dit :

- La nourriture est sortie de celui qui mange et du fort est venue la douceur.

Ils passèrent trois jours sans résoudre l'énigme. Vint le septième jour du festin sans qu'ils fussent plus heureux. Mais, ayant suborné la femme de Samson, les jeunes Philistins s'approchent de lui avant le coucher du soleil et lui demandent :

Quoi de plus doux que le miel ?

Quoi de plus fort que le lion?

- Si vous n'aviez pas labouré avec ma génisse, leur répond le jeune Israélite, dans ce style figuré qui avait le don d'exaspérer M. de Voltaire, vous n'auriez pas résolu mon problème.

Et soudain, pour acquitter sa dette de jeu, il descend vers Ascalon, décervèle trente Philistins, prend leurs dépouilles et les donne, comme habits de couleurs variées, aux jeunes hommes qui avaient répondu à sa question.

Le commerce des vêtements dé- 
fraîchis, des costumes hors d'usage et des redingotes d'uccasion était désormais fondé en Israël...

Salomon faisait entrer des abeilles dans son palais, pour discerner la rose véritable de la rose artificielle que Balkis, reine de l'Yemen, lui avait apportée afin d'éprouver sa sagesse.

Dans l'évangile selon Mathieu, Jésus mange avec ses apôtres un rayon de miel, ce qui permet aux théologiens d'affirmer, avec le sérieux des baudets qu'on étrille, que ce rayon, composé de miel et de cire, figure la double nature de Jésus, faite d'humanité et de divinité.

Dans les répons de la liturgie catholique, Cécile est qualifiée " apis argumentosa ", c'est-à-dire abeille pleine d'arguments. L'Eglise traduit par " abeille industrieuse "; mais c'est une interprétation évidem- 
ment erronée. En effet,Cécile,jeune, riche, patricienne, érudite et raisonneuse, discutant avec ses juges, prétendait les convaincre. Elle s'estimait leur égale, tenait tête à leurs objections. On n'a pas de peine à comprendre que le jeune Valère, époux d'une femme si "argumenteuse " et ferrée en théologie, ait acquiescé de grand cœur et tout de suite au vœu de chasteté dont il était requis.

"Les erreurs charmantes des anciens qui observaient la nature avec des yeux encore tout éblouis par la présence de dieux imaginaires » ont fourni aux hagiographes populaires un certain nombre de thèmes, dont quelques-uns gardent encore un parfum d'idylle antique et de noble poésie.

Au XII siècle, Pierre le Vénérable, abbé de Cluny, au XVIIe, le P. Pietra Santa (Thaumasia vere. 
religionis, I665, Rome) ont déduit le conte du Ciboire ou du Tabernacle de cire, légende immuable pour le fond, mais, quant à la forme, assez diaprée, et que les hagiographes modernes, abbé Postel, P. Giry, etc. ont affadie extraordinairement de mucilages sulpiciens.

Jacques de Voragine et les Bollandistes aprèslui ont négligé ce miracle dont les éleveurs d'abeilles eussent pu faire un tableau corporatif au beau temps des jurandes, maîtrises et corps de métiers.

Pénétrant, une nuit, dans je ne sais plus quelle église de Bohême, des malfaiteurs dérobaient des ornements et les vases sacrés, chasubles, dalmatiques, étoles de drap d'or et surplis de dentelle. Toutes les magnificences du chapier, les trésors de la sacristie étaient déjà tombés entre leurs mains scélérates. Mais 
l'un d'eux, plus hardi ou plus avide, força le tabernacle et larronna le ciboire contenant les hosties consacrées. Epouvantés, ses compagnons prirent la fuite. Alors, moitié pour les rassurer, moitié pour ne charger point sa conscience d'un forfait improductif, le mauvais garçon, en gagnant au pied, jeta les saintes espèces non loin du rucher qu'entretenait le curé du lieu. A l'aube du matin, le clergé, terrifié, constatait avec horreur le larcin et le sacrilège. Puis, ce fut le tour des personnes pieuses qui toutes, à grands cris et de tous côtés, se mirent au pourchas des malfaiteurs. Soudain, un enfant de douze ans, renommé pour la douceur et la piété de son âme, entendit, près des ruches, un murmure inusité. Il approche. O miracle! dans un ostensoir de cire vierge, resplendissaient, intactes, les parcelles abandonnées sur le chemin, 
tandis que les insectes, autour d'elles, bourdonnaient un cantique d'amour dans le soleil levant.

Tolstoï, dans le mythe des Deux Vieillards fait intervenir les abeilles en un récit digne d'être conté par les rédacteurs de la Légende Dorée.

L'un et l'autre vieillard ont fait le vœu d'un pélerinage en TerreSainte. Ils partent de conserve. Mais voici que l'un d'eux, petit homme jovial, grand buveur et même un peu ivrogne, quitransforme volontiers en wodka le miel de ses abeilles, s'arrête, à quelques verstes de son village, pour accomplir les œuvres de miséricorde chez de tristes paysans que la famine a visités. L'autre, plus littéral, strict observateur des engagements pris, ne s'arrête guère et poursuit le voyage. Arrivé dans Jérusalem, il gagne le Saint-Sépulcre et, parmi la foule, assiste à l'office du matin. Or, devant le 
maître-autel, sous un rayon de soleil qui l'enveloppe comme le nimbe d'une icône, il aperçoit, transfiguré, le compagnon quitté làbas, dans le désert. Des abeilles d'or voltigent autour de sa barbe blonde, comme on en voit aux images de Dionysos, le Christ du paganisme, ou parfumant de leur ambroisie le banquet d'Agathon. Le vieillard, étonné, cherche partout son camarade et ne le peut trouver. Enfin, il reprend le chemin de la sainte Russie.

Un soir, à la nuit tombante, il atteint la demeure famélique où son compagnon et lui firent halte au départ. Tout en est changé; le travail a ramené la prospérité, la vigueur et la joie. Devant la porte, un enfant joue avec le petit vieillard qui s'arrêta pour secourir de pauvres gens.

- Mais, dit le pèlerin, je t'ai vu 
pourtant, agenouillé devant le SaintSépulcre ?

- Chut! dit l'autre. Ne parlons plus de ces choses.

Et le premier vieillard comprend enfin ceci : que les œuvres d'amour sont plus efficaces que les pratiques rituelles et que la Foi ne compte guère sans la Charité.

La Russie aime les abeilles. Nicolai Gogol met ses plus jolis contes dans la bouche de Roudiy Panko, l'éleveur d'abeilles de l'Ukraine.

Ronsard a interprété délicieusement l'épigramme de l'Anthologie où l'Amour piqué se plaint à sa inère du tort que lui fit la cuisante mouche qui visite les fleurs.

Les tristes vers de l'abbé Delille traduisent imparfaitement l'erreur de Virgile. Pour créer un essaim nouveau, le Mantouan conseille de tuer un bœuf et d'abandonner ses 
restes à la putréfaction dans un enclos parfumé de sauge, de mélilot, de verveine et de romarin.

... le berger dans ses nombreux troupeaux $\mathrm{Va}$ choisir à l'instant quatre jeunes taureaux, Inmole un nombre égal de génisses superbes Qui des prés émaillés foulaient en psix les herbes. Pour la neuvième fois quand l'aurore parut, Au malbeureux Orphée il offrit son tribut, Et rentra plein d'espoir dans la forêt profonde.

O prodige ! le sang par sa chaleur fẻconde, Dans le flanc des taureaux forme un nombreux essaim; Des peuples bourdonnants s'échappent de leur sein, Comme un nuage épais dans les airs se répandent Et sur l'arbre roisin en grappe se suspendent.

Michelet explique d'une façon ingénieuse el touchante l'erreur du grand poète. Dans ce chant, écrit pour Varus et deux fois sacré, par le malheur et l'amitié, il a confondu avec les troupeaux d'Aristée les insectes dorés qui vivent leur innocente vie, exempts de travail, après avoir traîné leur première existence dans les liquides effroyables de la putréfaction.

Il les a vues pour la première 
fois, ces abeilles innocentes, par un jour indécis d'octobre, sur les asters débiles et les rudes cyprès de la sépulture familiale.

Tous les modernes ont triomphé de lignorance de Virgile et de sa fable d'Aristée, qui tire la vie de la mort et fait naître ses abeilles du flanc des taureaux immolés, moi, je n'en ai jamais ri. Je sais, je sens, que toute parole de ce grand poète sacré a une valeur très-grave, une autorité que j'appellerais augurale et pontificale. Le quatrième livre des Géorgiques, spécialement, fut une œuvre sainte, sortie du plus profond du cœur.C'était un pieux hommage au malheur et à l'amitié, l'éloge d'un proscrit, de Gallus, le plus tendre ami de Virgile. Cet éloge fut effacé, sans doute, par le prudent Mécène. Et Virgile y substitua sa résurrection des abeilles, ce chant plein d'immortalité, qui, dans le mystère des transformations de la nature, contient notre meilleur espoir : Que la mort n'est pas une mort, mais une nouvelle vie commencée.

Aurait-il pris le vain plaisir de faire un conte populaire à ce lieu consacré du poème qu'avait occupé le nom d'un ani ? Je 
ne le croirai jamais. La fable, si c'en est une, a dû avoir quelque base sérieuse, un côté de vérité. Ce n'est pas ici le poète mondain, le chanteur urbain, comme Horace, l'élégant favori de Rome. Ce n'est pas l'improviseur charmant de la cour d'Auguste, le léger, l'indiscret Ovide, qui trahit les amours des dieux. Virgile est lenfant de la terre, la noble et candide figure du vieux paysan italique, religieux interrogateur, soigneux et naif interprète des secrets de la nature. Qu'il se soit trompé sur les mots, qu'il ait mal appliqué les noms, cela n'est pas impossible; mais pour les faits, c'est autre chose: ce quil dit, je crois qu'il l'a vu.

Un hasard me mit sur la voie. Le $2 S$ octobre $18 ; 6$, nous montions au cimetière du Père-Lachaise pour visiter avant l'hiver les sépultures de ma famille, la tombe qui réunit mon père et son petit-fils. Ce dernier né métait renu l'année méme qui terminait la première moitié de ce siècle, et je l'avais nommé Lazare dans mon espoir religieux du réveil des nations. J'avais cru voir sur son visage comme une lueur des pensées fortes et tendres qui me remplissaient le cœur à ce dernier moment de mon enseignement. Vanité de nos espérances! 
Cette fleur de mon automne, que j'aurais voulu animer de la vitalité puissante qui a commencé tard pour moi, elle disparut presque en naissant. Et il me fallut déposer mon enfant aux pieds de mon père, déji mort depuis quatre années. Deux cyprès que je plantai alors dans cette mauvaise terre d'argile n'en ont pas moins pris en si peu de temps une étonnante croissance. Deux fois, trois fois plus hauts que moi, ils dressent des branches vigoureuses d'un jeune et riche feuillage qui veut toujours pointer au ciel. Qu'on les baisse avec effort, clles se relèvent fières et fortes, vivantes d'une incroyable sève, comme si ces arbres avaient bu dans la terre ce que j'y mis, le cher tresor de mon passé et mon invincible espérance.

Au milieu de ces persées, montant la colline, avant d'arriver à la tombe qui est dans l'allée supérieure, je faisais cette observation, qu'ayant eu tant d'occasions de fréquenter ce beau et triste lieu, ayant été a un autre âge le plus assidu visiteur des morts, je n'avais presque jamais vu d'insectes au Père-Lachaise. At peine, au grand moment des fleurs, lorsque tout en est couvert et que même nombre de vieux tombeaux abandonnés sont comme englou- 
tis dans les roses, je n'ai pas remarqué que la vie animale $y$ abondât, comme elle fait ailleurs. Peu d'oiseaux, très-peu d'insectes. Pourquoi ? Je ne pourrais le dire.

En faisant cette réflexion, nous avions achevé de gravir la colline; nous étions devant la tombe. J'y trouvai avec admiration, le dirai-je ? avec une sorte de saisissement, un surprenant démenti à ce que je venais le dire.

Une vingtaine environ de très brillantes abeilles voletaient sur le jardinet, aussi étroit qu'un cercueil, dépouillé et pauvre de fleurs, attristé de la saison. Il ne restait guère dans tout le cimetière que les dernières fleurs d'automne, quelques défaillantes roses du Bengale, demi-effeuillées. Le lieu même où nous étions, plein de constructions nouvelles, de maçonnage et de plàtre, était une Arabie déserte. Sur la tombe enfin, il n'y avait, vers la tête du grand-père, que quelques blancs asters, fort pàles, et sur mon enfant les cyprès. II fallait bien que ces asters, dans ce mauvais sol argileux, nourris ou des souffles de l'air, ou des esprits de la terre, gardassent un peu de miel, puisque ces petites glaneuses $\mathrm{y}$ venaient récolter encore.

Je ne suis pas superstitieur. Je ne crois qu'ì un miracle, le miracle permanent de 
la Providence naturelle. J'éprouvai pourtant combien une vive surprise de cœur peut ébranler l'esprit. Je me sentis reconnaissant de voir les mystérieux petits êtres animer cette solitude, où moi-même, hélas! je viens rarement. L'entrainement croissant du travail où les jours poussent les jours, la famme haletante de cette forge où l'on forge de plus en plus vite, doutant si l'on vivra demain, tout cela nous tient plus loin des tombeaux que nous n'y fûmes aux temps rêveurs de la jeunesse. Je fus saisi de voir celles-ci me sunpléer, tenir ma place. En mon absence elles peuplaient, vivifiaient le lieu, consolaient mes morts, les réjouissaient peut-être. Mon père leur aurait souri aree sa bonté indulgente; elles auraient fait le bonheur, la première joie de mon enfant.

L'intérêt ne les nıenait guère. Il y avait si peu à prendre pour elles! Cependant, quand nous suspendimes aux cyprès des couronnes d'immortelles que nous apportions, elles eurent la curiosité d'aller voir si ces nouvelles fleurs avaient en elles quelque chose. La dure et piquante corolle les rebuta vite, et les renvoya aux asters fanés. J'en fus triste, et je leur dis : a Tard, bien tard, vous venez, amies, et sur la tombe du 
pauvre !... Que n'ai-je à vous récompenser d'un petit banquet d'amitié, qui vous solitienne et vous réchauffe aux premiers froids qui déjà soufflent sur ces hauteurs glaciales, exposées au vent du nord!"

Comme si elles m'avaient compris, leurs mouvements répliquèrent juste. J'en vis qui, de leurs petits bras, adroitement tournés en arrière, se frottaient le dos au soleil ; elles voulaient s'imbiber à fond de ce rayon tiède et s'en pénétrer. Elles profitaient de l'heure malheureusement bien courte où le soleil tourne si vite; on le sent à peine, et il est passé. Leur geste, très significatif, disait manifesterent : "Oh! la froide matinée que nous avons eue !... Hâtonsnous! Avant une heure commence la soirée non moins froide, la nuit glacée, qui sait? l'hiver ! et bientôt la mort pour nous. "

Elles étaient très vives encore, merveilleusement propres et nettes, je dirai presque lumineuses, sous leurs ailes lustrées, glacées d'or. Je ne vis jamais de plus beaux insectes, plus visiblement animés d'une vie supérieure. Une chose m'embarrassait, c'est qu'elles étaient trop belles, trop luisantes, n'ayant point leur costume industriel, leur habit velu, leurs pinceaux, leurs brosses. Enfin, j'aperçus une chose, c'est qu'elles 
n'avaient pas non plus les quatre ailes de l'abeille, mais seulement deux.

Je reconnus mon erreur. Celles-ci sont justement celles qui trompèrent aussi Virgile. Comme moi, il les crut abeilles et leur a donné ce faux nom Réaumur avoue que lui-même il y fût un moment trompé.

Mais le fait conté par Virgile n'est pas inexact. On comprend qu'il ait vivement ému l'antiquité et qu'elle y ait vu un type de résurrection. Elles semblent les filles de la mort. Des trois âges de leur existence, elles passent le premier dans les eaux morbides et mortelles, funestes à tous les autres êtres, que laissent échapper les résidus de la vie en dissolution; par une tendresse ingénieuse, la nature les y préserve, les maintient vivantes et les fait respirer en pleine mort. Le second âge, elles le passent sous la terre, dans les ténèbres, pour y dormir leur sommeil de chrysalide. Mais, quittes de cette sépulture, elles sont bien dédommagées de leur abaissement antérieur; une vie légére, aérienne, exempte des travaux de l'abeille, glorifiée par des ailes d'or, comme celle-ci n'en eut jamais, leur est accordé, avec des mœurs douces. Innocentes et sans aiguillon, elles vivent leur saison d'amour sous le soleil et dans les fleurs. 
Loin de rougir de leur origine, nobles abeilles virgiliennes, elles ne dédaignent pas les fleurs du cimetière, elles font société aux morts, et, pour les vivants, recueillent ce miel de l'àme, l'espoir de l'avenir.

L'Empire choisit pour blason les abeilles, assez semblables aux fleurs delys propres à la maison de France; Napoléon porta le manteauimpérial de sinople aux abeilles d'or.

M. Freppel, évêque d'Angers, ennemi personnel de Gambetta, et qui fut à la Chambre, après la guerre, un des lutteurs les plus autorisés dans le " parti du boucan», suivit cet exemple auguste. Il se donna pour armoiries: d'azur aux trois abeilles d'or, avec la devise:

\section{Sponte favos, ægre spiculum,}

De grand cour les rayons, à regret la blessure.

Pour un homme aussi acariâtre, c'était là, peut-on dire, un programme bien melliflu.

Les abeilles du manteau impérial 
ont inspiré à l'auteur des Chdtiments l'un de ces morceaux à la fois sublimes et cocasses qui justifient le surnom de "Jocrisse à Pathmos » dont Barbey d'Aurévilly l'avait gratifié. Quand on relit, après cinquante ans, ses invectives contre Napoléon III, qui fut un homme de cœur et le modèle des gentlemen, on comprend quelle déchéance frappe l'artiste quand il quitte son pur domaine, descend parmi les rumeurs de la place publique, pour y mêler sa voix aux cris de la plèbe immonde et fraterniser avec les tueurs de dieux.

Ainsi les abeilles nourricières délectent les hommes de leur miel, de leur sagesse et de leur beauté. Ces guerrières symbolisent les arts de la paix, le culte du foyer. Elles montrent l'exemple des plus hautes vertus. Elles enseignent à l'homme le travail et le désintéressement, la 
concorde et la frugalité. Elles proposent à son humeur inquiète leur fidèle attachement à la règle des aieux.

Pour accoiter la douleur inévitable, pour expliquer notre raison d'être, nous conformer à la Loi du Monde, pour, sans amertume, accepter la vieillesse et la mort, elles apprennent à l'individu que le meilleurstratagème est de vouerses jours à quelque sublime entreprise, d'aimer en dehors de soi-même et, pour une œuvre collective, de rechercher dansson existence éphémère un principe d'immortalité.

At genus immoriale manct multosque per annos Stat fortuna domus et avi numerantur avorum.

21 février 1907. 



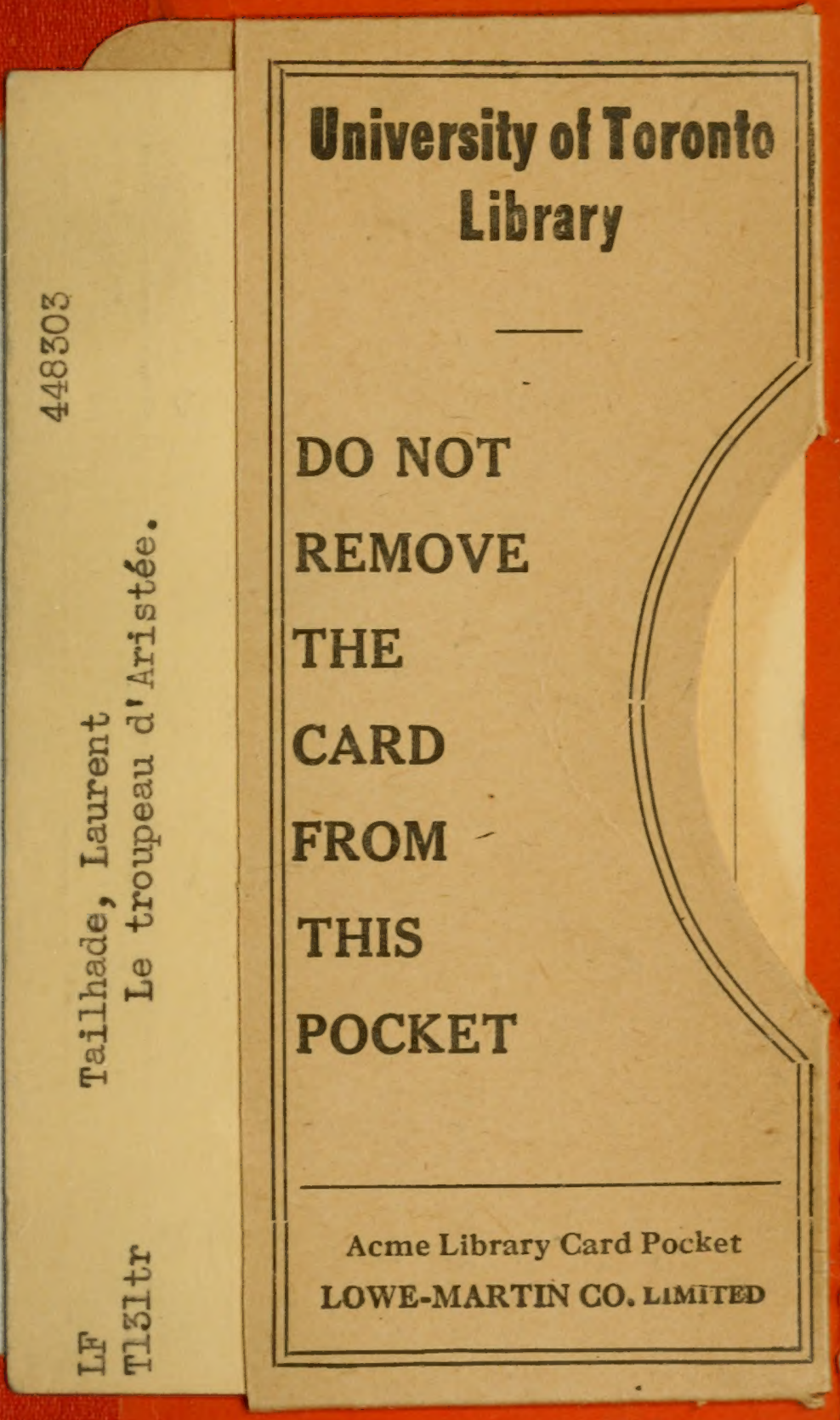




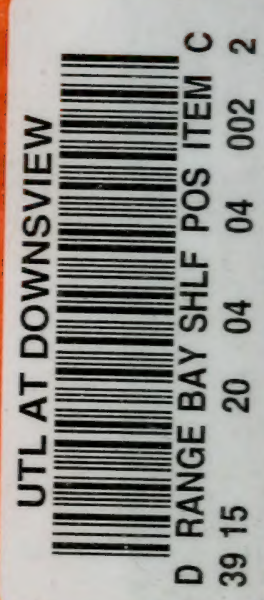

